Research Paper

\title{
Inter- and intra-observer reproducibility of ADC measurements in esophageal carcinoma primary tumors
}

\author{
Zhimin $\mathrm{Ye}^{1, *}$, Jun Fang ${ }^{1, *}$, Shujun $\mathrm{Dai}^{2}$, Tieming $\mathrm{Xie}^{3}$, Fangzheng Wang ${ }^{1}$, Zhun \\ Wang $^{1}$, Kai $\mathrm{Li}^{3}$, Zhenfu Fu ${ }^{1}$ and Yuezhen Wang ${ }^{1}$ \\ ${ }^{1}$ Department of Radiation Oncology, Zhejiang Cancer Hospital, Hangzhou, China \\ ${ }^{2}$ Department of Intensive Care Unit, The Second Affiliated Hospital, Zhejiang University School of Medicine, Hangzhou, China \\ ${ }^{3}$ Department of Radiology, Zhejiang Cancer Hospital, Hangzhou, China \\ *These authors are considered as co-first authors \\ Correspondence to: Zhun Wang, email: wangzhun007@hotmail.com \\ Zhenfu Fu, email: fuzf1000@163.com \\ Keywords: esophageal carcinoma, diffuse weighted imaging, apparent diffused coefficient, reproducibility \\ Received: June 16, $2017 \quad$ Accepted: August 17, $2017 \quad$ Published: October 06, 2017 \\ Copyright: Ye et al. This is an open-access article distributed under the terms of the Creative Commons Attribution License 3.0 \\ (CC BY 3.0), which permits unrestricted use, distribution, and reproduction in any medium, provided the original author and source \\ are credited.
}

\section{ABSTRACT}

The apparent diffuse coefficient (ADC) may correlate with the treatment response to chemotherapy/radiotherapy in solid tumors. Our aim was to determine the inter- and intra-observer reproducibility of ADC measurements in primary esophageal squamous cell carcinoma (ESCC). ADCs were blindly measured in 31 patients diagnosed with ESCC by two observers before treatment (pre-ADC) and after $5^{\text {th }}$ fraction radiotherapy (intra-ADC) twice with a 2-week interval. The mean pre-ADC of primary tumors was $1.25 \pm 0.22$ and $1.27 \pm 0.23$ (in $10^{-3} \mathrm{~mm}^{2} / \mathrm{s}$ ) from observer $A$ for measurements 1 and 2, respectively, and the intra-observer measurements were $\mathbf{- 0 . 0 2}$ bias vs. $\mathbf{- 0 . 1 3 - 0 . 0 9}$ limits of agreement. From observer $B$, the mean pre-ADC varied between $1.25 \pm 0.23$ and $1.27 \pm 0.23$ (in $10^{-3} \mathrm{~mm}^{2} / \mathrm{s}$ ) for measurements 1 and 2 , respectively, and intraobserver measurements were -0.02 bias vs. $-0.17 \sim 0.16$ limits of agreement. The mean pre-ADC of primary tumors was $1.26 \pm 0.24$ (in $10^{-3} \mathrm{~mm}^{2} / \mathrm{s}$ ) from observers $A$ and $B$, and inter-observer measurements were 0.01 bias vs. $-0.09-0.09$ limits of agreement, revealing a low inter-observer variance. Similar measurements of the intra-SD parameters showed that the pre- and intra-ADC of primary tumors differed significantly. Thus ADC measurements may have sufficient inter-observer and intraobserver reproducibility to measure primary tumor responses to treatment, and the ADCs before and during treatment differed.

\section{INTRODUCTION}

Treatment response of esophageal carcinoma (EC) is affected by many factors including oxygenation status of cancer cells, gene mutation, and distribution of microvascular vessels [1-3]. There is also variability in radiotherapy dosage among different treatment centers. For example, the RTOG 94-05 phase III trials demonstrated that the survival or local/regional control in the group of higher radiation dose $(64,8 \mathrm{~Gy})$ was not increased compared with that in the group of lower radiation dose (50.4Gy) [4]. Moreover, in the CROSS phase III trial, disease-free survival (DFS) and overall survival were improved in patients underwent radiation doses of 41.4Gy preoperative chemoradiotherapy (CRT) compared to patients that underwent surgery alone [5]. If the sensitivity of chemotherapy/radiotherapy response is monitored early, the effectiveness of these treatment regimens will be better predicted.

Diffusion-weighted imaging (DWI) is a functional approach that detects water molecule diffusion in the body, and the apparent diffusion coefficient (ADC) has 
Table 1: General clinical characteristics of study population $(n=31)$

\begin{tabular}{lc}
\hline Character & No \\
\hline Gender & 20 \\
M & 11 \\
F & $64.5 \pm 8.7$ \\
Age (years) & \\
PS & 3 \\
0 & 16 \\
1 & 12 \\
2 & \\
T stage & 0 \\
T1 & 3 \\
T2 & 24 \\
T3 & 4 \\
T4 & \\
Location of tumor & 3 \\
Neck & 4 \\
Upper thoracic & 24 \\
Middle thoracic & 17 \\
Lower thoracic & 7 \\
Chemotherapy & $200.65 \pm 647.94$ \\
Neo-adjuvant &
\end{tabular}

been utilized for the clinical application of evaluating the treatment response to CRT in many cancers [6]. Clinically valid use of DWI requires that measurement variation in a given patient be less than that observed by different observers or measurement. The inter- and intrareproducibility of $\mathrm{ADC}$ measurement is rarely reported for primary tumors of EC patients, and contouring of the measurement region of interest (ROI) is not standardized. The variation of protocols, b-values, and calculations reported by different institutions to obtain the ADC values and cut-off values cannot be compared and are not possible to utilize clinically $[7,8]$. Therefore, the broad application of DWI in the prediction of treatment response is dependent on the accuracy and reproducibility of the measurements.

We estimated the reproducibility of two measurements of ADC (at baseline and the $5^{\text {th }}$ fraction of RT) via a designated method and explored the change in ADC during the early stages of treatment.

\section{RESULTS}

\section{General clinical data of 31 patients}

A total of 31 patients (20 men, 11 women; mean age $64.5 \pm 8.7$ years) were diagnosed with esophageal squamous cell carcinoma (ESCC). The number of patients in each T stage were $\mathrm{T} 1, \mathrm{n}=0, \mathrm{~T} 2, \mathrm{n}=3, \mathrm{~T} 3, \mathrm{n}=24$, T4, $\mathrm{n}=4$, respectively. Three cases scored performance status (PS) of 0,16 cases scored PS of 1 , and 12 cases scored PS of 2. Primary tumor sites were located in the neck of 3 cases, upper thoracic of 4 cases, middle thoracic of 17 cases, and lower thoracic of 7 cases. The mean RT dose was $5800.65 \pm 647.94 \mathrm{cGy}$.

\section{Measurement and reproducibility of ADC and SD before treatment}

Before treatment, mean pre-ADC of primary tumors were $1.25 \pm 0.22$ and $1.27 \pm 0.23$ (in $10^{-3} \mathrm{~mm}^{2} / \mathrm{s}$ ) from 
Table 2: Twice measurements of pre-ADC in observer A and B

\begin{tabular}{lcc}
\hline Observer & Measurement & Mean pre-ADC (in $\left.\mathbf{~ 1 0}^{-3} \mathbf{m m}^{2} / \mathbf{s}\right)$ \\
\hline A & 1 & $1.25 \pm 0.22$ \\
& 2 & $1.27 \pm 0.23$ \\
B & 1 & $1.25 \pm 0.23$ \\
& 2 & $1.27 \pm 0.23$ \\
\hline
\end{tabular}

Table 3: Twice measurements of pre-SD in observer A and B

\begin{tabular}{lcc}
\hline Observer & Measurement & Mean pre-SD (in $\left.\mathbf{~ 1 0}^{-3} \mathbf{m m}^{2} \mathbf{s}\right)$ \\
\hline A & 1 & $0.24 \pm 0.05$ \\
& 2 & $0.25 \pm 0.05$ \\
B & 1 & $0.22 \pm 0.05$ \\
& 2 & $0.24 \pm 0.05$ \\
\hline
\end{tabular}

observer A for the measurement 1 and 2 respectively, and $1.25 \pm 0.23,1.27 \pm 0.23$ from observer $\mathrm{B}$, respectively (Table 2). The intra-observer measurements were -0.02 bias vs. -0.13 0.09 limits of agreement from observer A and -0.02 vs. $-0.17 \sim 0.16$, respectively, from observer B (Figure 1A, 2A). The mean pre-SD of primary tumors for measurement 1 and 2, respectively, were $0.24 \pm 0.05$ and $0.25 \pm 0.05$ (in $10^{-3} \mathrm{~mm}^{2} / \mathrm{s}$ ) from observer $A$ and $0.22 \pm 0.05$, $0.24 \pm 0.05$, from observer B (Table 3). The intra-observer measurements were -0.01 bias vs. $-0.06 \sim 0.05$ limits of agreement from observer $\mathrm{A}$ and -0.02 vs. $-0.11 \sim 0.08$, respectively from observer $\mathrm{B}$ (Figure 1B, 2B). The interobserver measurements in pre-ADC and pre-SD were 0.01 bias vs. $-0.09 \sim 0.10$ limits of agreement and $0.01 \mathrm{vs}$. $-0.07 \sim 0.09$, respectively (Figure 3A, 3C).

\section{Measurement and reproducibility of ADC and SD during treatment}

At the $5^{\text {th }} \mathrm{RT}$, the mean intra-ADC of primary tumors from observer A was $1.57 \pm 0.32$ and $1.59 \pm 0.30$ (in $10^{-3} \mathrm{~mm}^{2} / \mathrm{s}$ ) for measurement 1 and 2 respectively, and $1.60 \pm 0.34,1.58 \pm 0.33$ from observer B (Table 4). The bias vs. limits of agreement for the intra-observer measurements corresponding to Bland-Altman plots from observer $\mathrm{A}$ and $\mathrm{B}$ are displayed in Figure 1C, $2 \mathrm{C}$, respectively. The inter-observer measurements of intra-ADC were -0.03 bias vs. $-0.15 \sim 0.10$ limits of agreement (Figure 3B). The mean intra-SD of primary tumors from observer A and B is summarized in Table 5 for measurement 1 and 2 , and the intra-observer bias vs. limits of agreement are displayed in Figure 1D, 2D. The inter-observer bias vs. limits of agreement for the
intra-ADC and intra-SD are shown in Figure 3B, 3D, respectively.

\section{Differential analysis of ADC and SD measurements}

Compared to the value of pre-ADC, the value of intra-ADC was significantly higher $(\mathrm{P}<0.05$, Figure $4 \mathrm{~A})$. However, while the value of intra-SD was higher than pre$\mathrm{SD}$, there is no significant difference between pre-SD and intra-SD ( $\mathrm{P}>0.05$, Figure 4B).

\section{DISCUSSION}

Functional imaging such as DWI is increasingly prominent in the treatment response evaluation of esophageal carcinoma due to the recent widespread application of MR for esophagus examination. However, a major challenge to the interpretation of functional metabolic imaging-generated parameters, including the ADC value of DWI, is the inherent physiologic heterogeneity within a tumor. To our best of knowledge, there is no standard protocol for performing ADC measurements of esophageal carcinoma.

Notably, few published studies have investigated the clinical value of DWI in evaluating esophageal carcinoma. Several studies have used "whole tumor" ROI data to differentiate malignant and benign nodes in esophageal carcinoma $[10,11]$ and predict RT response $[12,13]$. Some groups have advocated assessment of only the most enhanced voxels within a tumor, based on the result that the most enhanced ROIs provided more statistically significant differences between responders and non-responders in CRT than whole tumor ROI [14]. Many 
Table 4: Twice measurements of intra-ADC in observer $\mathrm{A}$ and $\mathrm{B}$

\begin{tabular}{lcc}
\hline Observer & Measurement & Mean intra-ADC (in $\left.\mathbf{~ 1 0}^{-\mathbf{3}} \mathbf{m m}^{\mathbf{2}} / \mathbf{s}\right)$ \\
\hline $\mathrm{A}$ & 1 & $1.57 \pm 0.32$ \\
& 2 & $1.59 \pm 0.30$ \\
$\mathrm{~B}$ & 1 & $1.60 \pm 0.34$ \\
& 2 & $1.58 \pm 0.33$ \\
\hline
\end{tabular}

Table 5: Twice measurements of intra-SD in observer A and B

\begin{tabular}{lcc}
\hline Observer & Measurement & Mean intra-SD $\left(\right.$ in $\left.\mathbf{~ 1 0}^{-3} \mathbf{m m}^{2} / \mathbf{s}\right)$ \\
\hline A & 1 & $0.26 \pm 0.08$ \\
& 2 & $0.28 \pm 0.07$ \\
B & 1 & $0.25 \pm 0.07$ \\
& 2 & $0.26 \pm 0.07$ \\
\hline
\end{tabular}
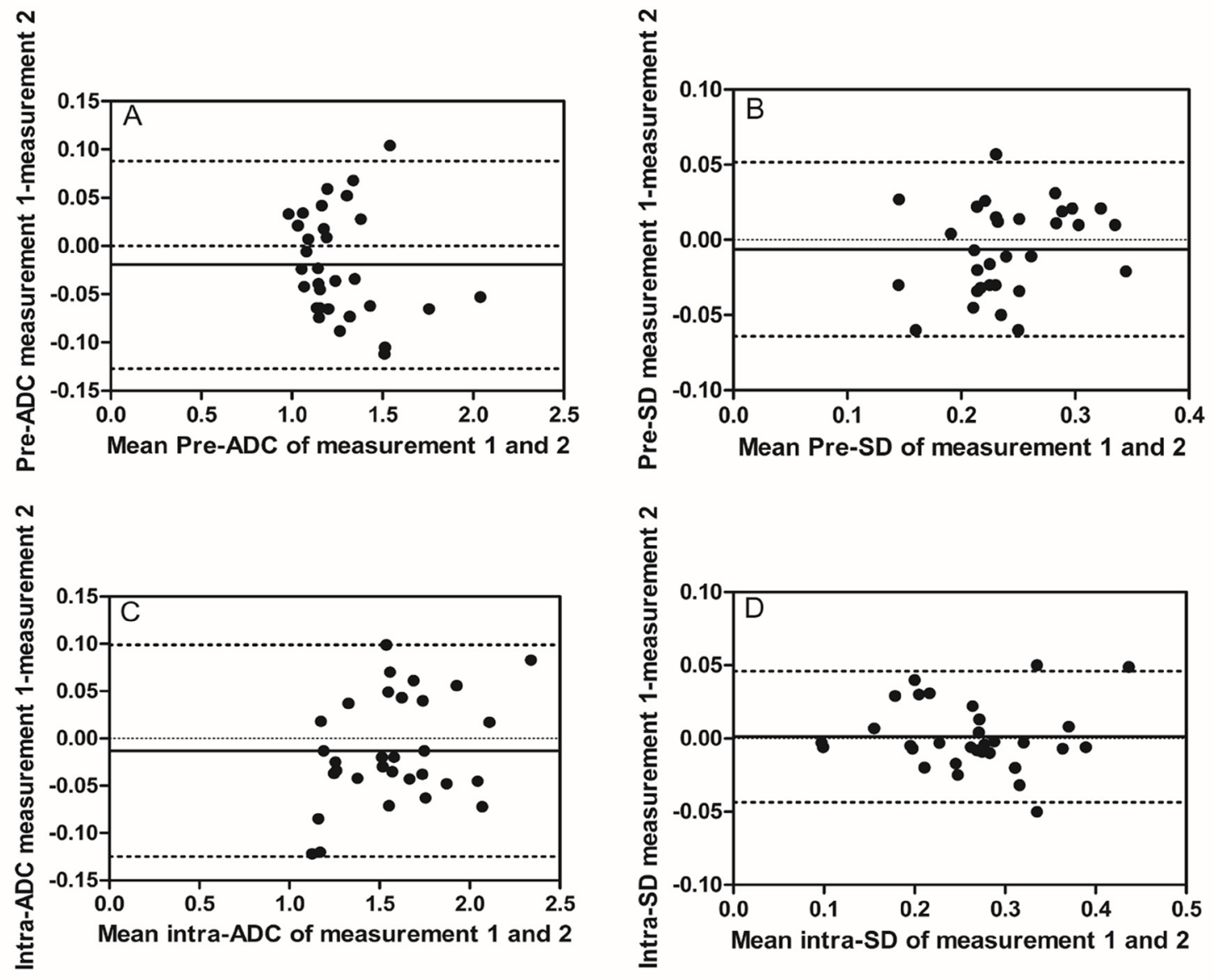

Figure 1: Intra-observer reproducibility of primary tumor ADC and SD measurements from observer A. Bland-Altman plots of difference of ADC or SD measurements (y-axis) vs. mean ADC or SD measurement (x-axis), with mean absolute difference (bias) (continuous line) and 95\% confidence interval (CI) of the mean difference (limits of agreement) (dashed lines except zero line). (A) The measurement of pre-ADC in primary tumor, (B) the measurement of pre-SD in primary tumors, (C) the measurement of intra-ADC in primary tumors, and (D) the measurement of intra-SD in primary tumors. The results showed relatively good intra-observer reproducibility with most plots distributed within the 95\%CI. 
studies neglect to illustrate the delineation of ROI and do not report intra- and inter-observer reproducibility of the ADC measurement $[15,16]$.

Our study specifically addressed ROI selection strategies to estimate intra- and inter-observer reproducibility. The method of ROI contouring in our study relied on the following strategies: (1) ROI in the slice containing the most enhanced voxels in enhanced contrast T1WI and excluded the necrotic areas to avoid intra-tumoral variation $[9,17,18]$, and the point was widely recommended for the measurement of ADC; and (2) Selection of three continuous sections, including the largest slice, to determine the average ADC of the tumor (Figure 5). Our method was derived from previous studies
$[19,20]$ where the delineation was based on the largest slice, but the ROI our study was not confined to the largest slice to assure low variance during the period of ROI delineation. Our data suggest that this is an appropriate strategy to assure the reproducibility of intra-observer and inter-observer. Furthermore, the resulting bias and limits of agreement measurements were acceptable, and low variance in ADC measurements was indicated by the parameter SD. Our results were consistent with Kwee et al. [21] who determined that semi-automated volumetric ADC measurements were more reproducible than manual ADC measurementxoldaxas. However, Kwee et al. [22] revealed that despite good inter- and intraobserver reproducibilities, the ADC value was not always
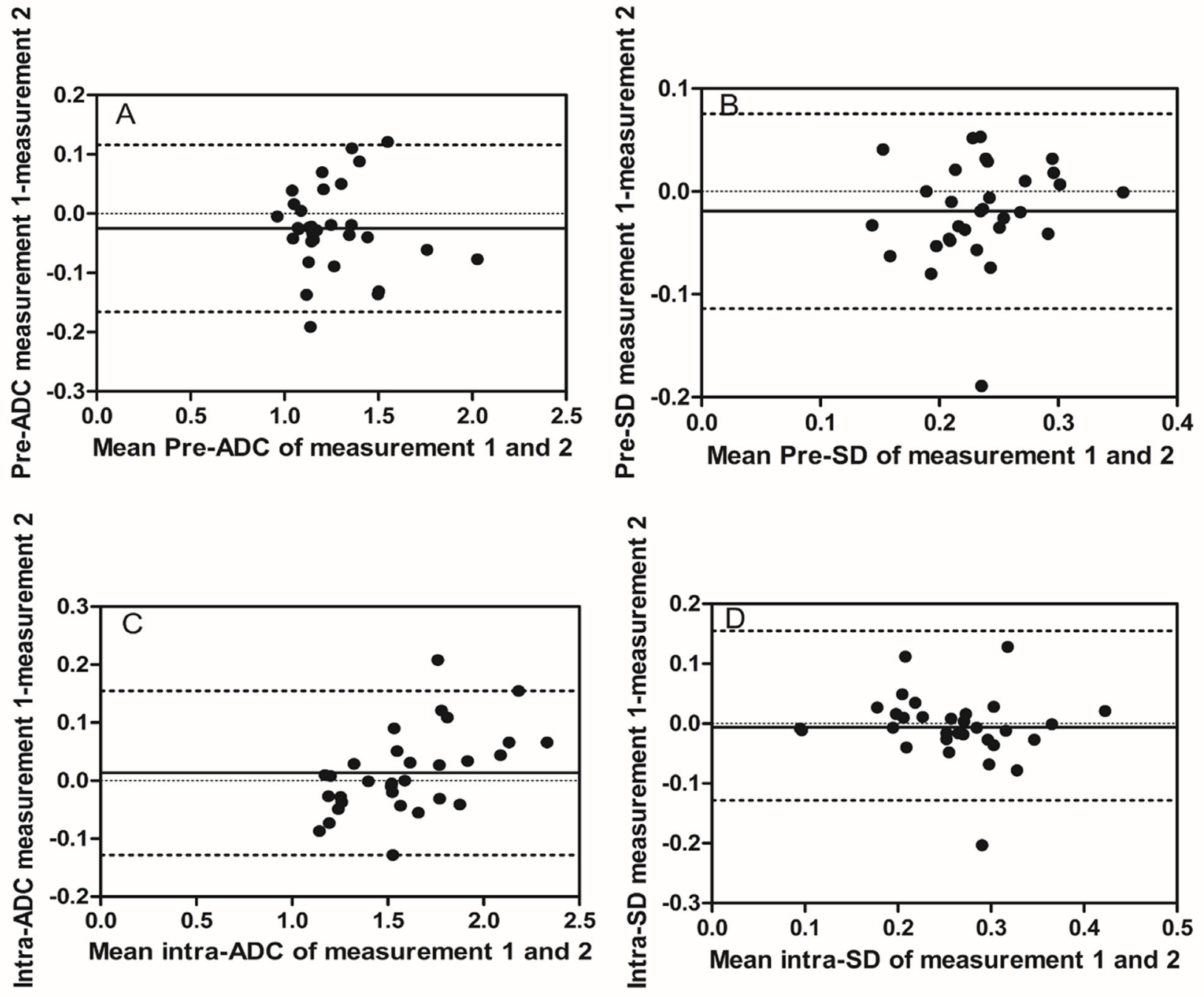

Figure 2: Intra-observer reproducibility of primary tumor ADC and SD measurements from observer B. Bland-Altman plots of difference of ADC or SD measurements (y-axis) vs. mean ADC or SD measurement (x-axis), with mean absolute difference (bias) (continuous line) and 95\% confidence interval of the mean difference (limits of agreement) (dashed lines except zero line). (A) The measurement of pre-ADC in primary tumors, (B) the measurement of pre-SD in primary tumors, (C) the measurement of intra-ADC in primary tumors, and (D) the measurement of intra-SD in primary tumors. 

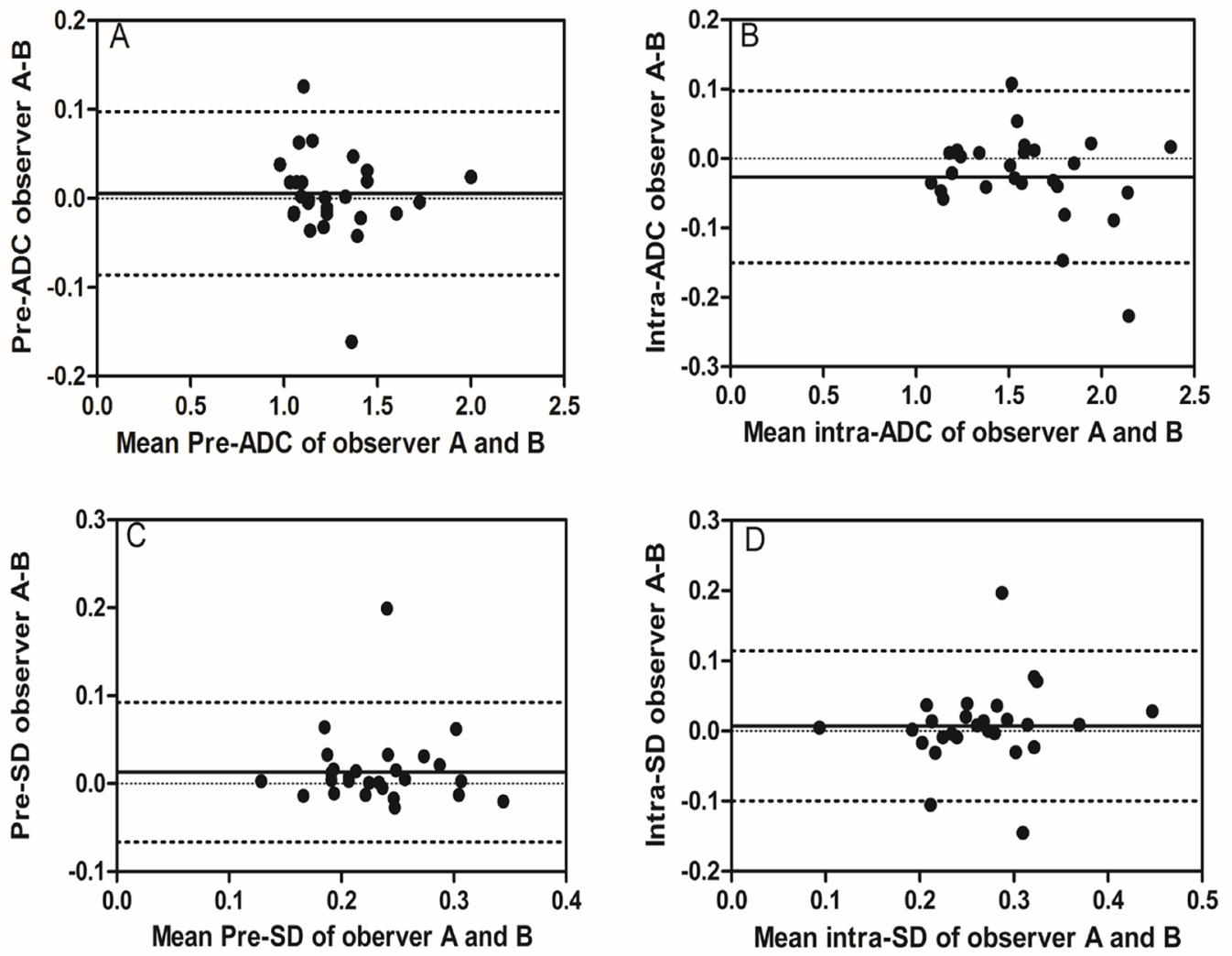

Figure 3: Inter-observer reproducibility of DWI primary tumor ADC measurements from observer A and B. BlandAltman plots of difference of ADC or SD measurements (y-axis) vs. mean ADC measurement (x-axis), with mean absolute difference (bias) (continuous line) and 95\% confidence interval (CI) of the mean difference (limits of agreement) (dashed lines except zero line). The results showed that inter-observer reproducibility was acceptable, which displayed most plots distributed between the lines of 95\% CI. (A) The measurement of pre-ADC between observer A and B, (B) the measurement of intra-ADC between observer A and B, (C) the measurement of pre-SD between observer A and B, and (D) the measurement of intra-SD between observer A and B.

A

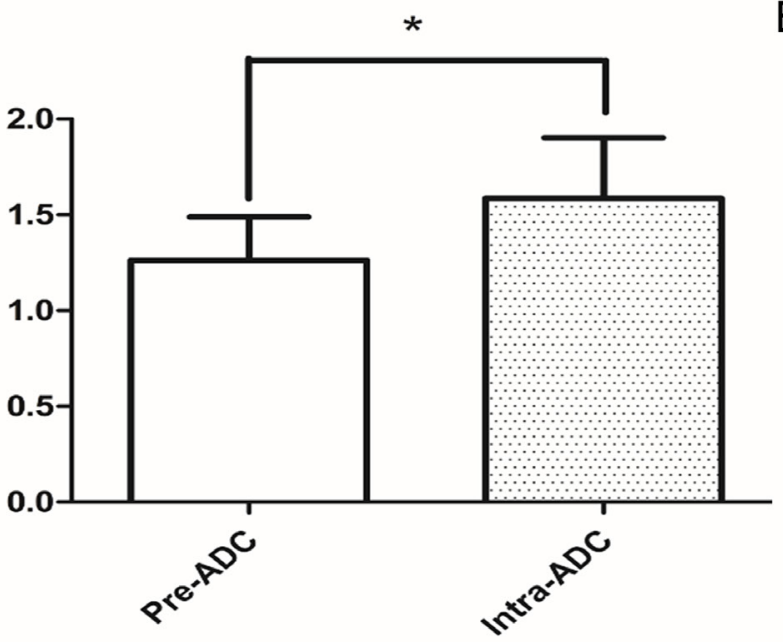

B

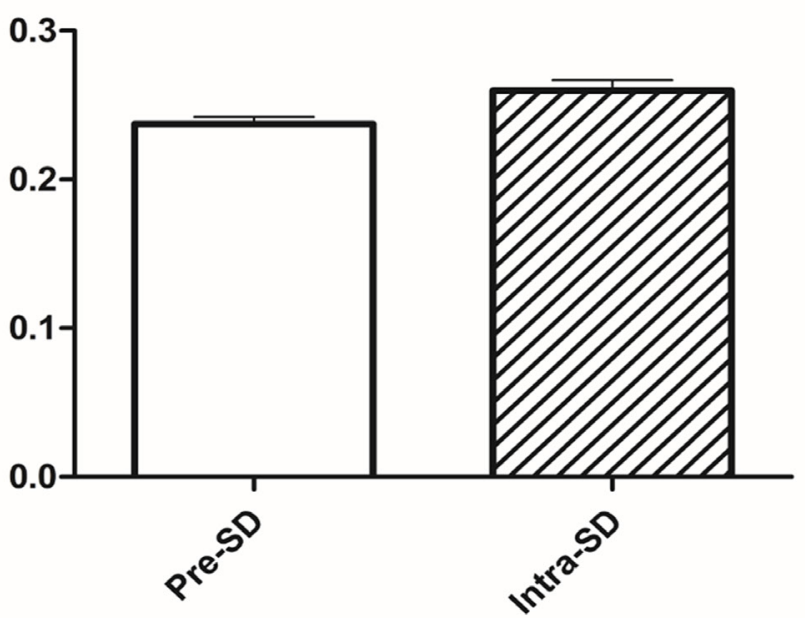

Figure 4: The comparison of ADC and SD parameters between pre-treatment and the $5^{\text {th }}$ RT. (A) The value of intra-ADC was higher than that of pre-ADC ( $(* \mathrm{P}<0.05)$; (B) the value of intra-SD was higher than that of pre-SD, but the difference was not statistically significant $(\mathrm{P}>0.05)$. 
sufficiently reproducible to discriminate malignant from non-malignant lymph nodes.

Interestingly, we also found that the ADC was significantly different at $5^{\text {th }}$ fraction RT which showed the change of functional parameters preceded the change of anatomical morphology. This result suggests the potential of ADC to predict the treatment response of esophageal carcinoma earlier. The check-point of monitoring response may shift earlier to avoid interference from tumor reduction that causes measurement error. The optimal check-point of treatment response is still controversial [8, $14,15]$, so the method in our study may be an alternative to monitoring early treatment response. Studies are ongoing in our center.

In conclusion, the ADC measurement from DWI is highly reproducible in esophageal carcinoma via our method and could predict treatment response.

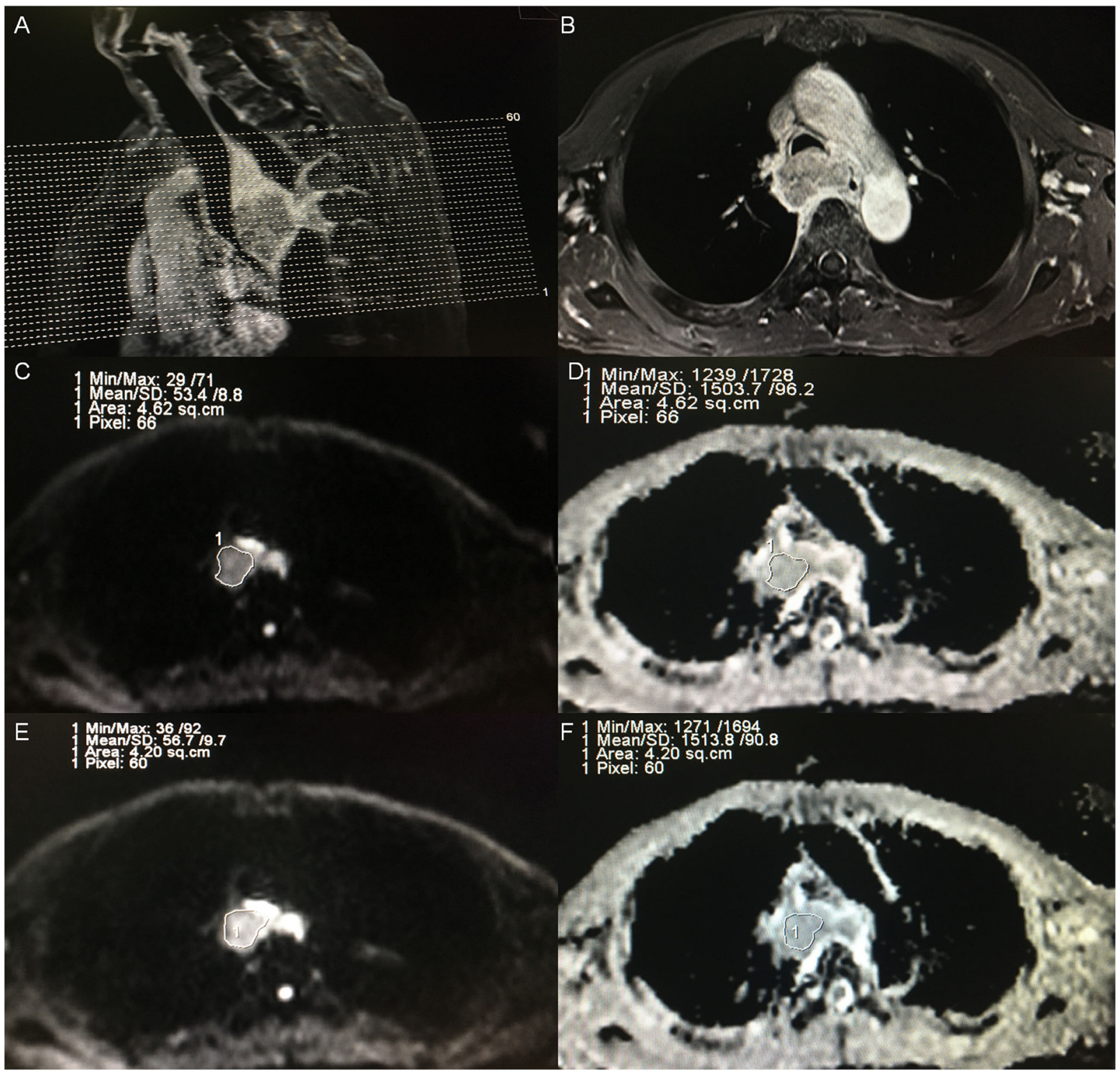

Figure 5: Example of ADC measurements of a primary tumor in a patient diagnosed as esophageal carcinoma before treatment. (A) A sagittal view with position line of $\mathrm{T} 1$-weighted plus contrast-enhanced $(\mathrm{T} 1+\mathrm{C})$ image for the primary tumor. (B) A transverse view of $\mathrm{T} 1+\mathrm{C}$ image for the one of three continuous sections with maximal diameter of tumor according to the sagittal and transverse view. (C) A region of interest (ROI) was placed manually for observer A in the selected section, on the image obtained at a b-value of $1000 \mathrm{~s} / \mathrm{mm}^{2}$, and the ROI was then copied and pasted onto the ADC map (D), and the ADC and SD of the selected section were automatically calculated. (E) A region of interest (ROI) was placed manually for observer B in the selected section, on the image obtained at a b-value of $1000 \mathrm{~s} / \mathrm{mm}^{2}$, and the ROI was then copied and pasted onto the ADC map (F), and the ADC and SD were also automatically calculated. 


\section{MATERIALS AND METHODS}

\section{Patient selection}

Thirty-one patients (20 men, 11 women; mean age 64.5 \pm 8.7 years; age range 41-79 years) diagnosed with ESCC by pathology, and treated at Zhejiang provincial cancer hospital between January 2015 and November 2016 were enrolled in this study. The study was approved by the institutional review board, and written informed consent was obtained from each participant before MRI examination. All subjects were qualified by the following criteria: (1) Eastern Cooperative Oncology Group performance status score is smaller than or equal to 2; (2) Adequate organ function; (3) No concomitant malignancy; (4) Good compliance; (5) No contraindication to MRI examination; (6) No surgical indications or patient refusal; (7) and completion of the entire course of radiotherapy. The stage of disease was classified according to the 7th edition of the Union for International Cancer Control (UICC) and the American Joint Committee on Cancer (AJCC) staging system. The clinical characteristics of all patients are listed in Table 1.

\section{MR imaging}

All subjects underwent two MRI examinations with a 1.5-T MR scanner (Achieva, Philips Medical Systems, Best, The Netherlands) using a phased array body coil (SENSE body coil, Philips Medical Systems, Best, The Netherlands). Examinations were performed before the initiation of treatment and at the $5^{\text {th }}$ fraction radiotherapy (RT) point. All MRI examinations contained axial spinecho T1 weighted imaging (TR/TE 423/100 ms, average number 1, FOV $365 \times 284 \mathrm{~mm}$, matrix 320 , slice thickness $4 \mathrm{~mm}$, skip 1.2-1.6 $\mathrm{mm}$ and slice 20), axial turbo spinecho T2 weighted imaging (TR/TE 2,000/70 ms, flip angle $180^{\circ}$, concatenations 2 , average number 2 , FOV $300 \times 280$ $\mathrm{mm}$, matrix 288 , slice thickness $4 \mathrm{~mm}$, skip $1.2-1.6 \mathrm{~mm}$ and slice 20), T1 with contrast enhanced imaging including sagittal and transverse axial, and then DWI (TR/TE/TI $10,205 / 70 / 180 \mathrm{~ms}$, FOV $450 \times 366 \mathrm{~mm}$, matrix 256 , slice thickness/gap 4/0 mm, slice 20, EPI factor (echo train length) 43). DWI scans were obtained using a single-shot spin-echo type of echo-planar sequence, and fat signals were suppressed using short-tau inversion recovery (STIR). The DWI $b$-values were $b=0$ and $1,000 \mathrm{~s} / \mathrm{mm}^{2}$. An interval of 7 min was allocated to acquire DWI with free breathing.

\section{Imaging analysis}

Both MR images were transferred to a workstation (ViewForum; Philips Medical Systems, Best, The Netherlands). Two board-certified radiologists (observer 1, Tieming Xie, with 13 years of experience in MR imaging; observer 2, Mingxiang Jiang, with 12 years of experience in MR imaging) reviewed the images and recorded the locations and slice numbers of the primary tumor site independently and blindly, and then performed ADC measurements of the selected tumor through the contouring region of interest (ROI). Each ROI was variable so that the two observers obeyed the following stipulations: (1) Used ROI in the slice containing the most enhancing voxels in enhanced contrast T1WI [9]. (2) Avoided the non-enhancement or necrotic areas in the ROIs. (3) Selected three continuous slices including the one of maximal diameter and its adjacent above and below one in tumor parenchyma according to the sagittal and horizontal view, and the values of ADC were averaged based on the three slices. (4) All measurements were performed twice by each observer, with a wash-out period of at least two weeks between the first and second series of measurements. The pre-treatment and $5^{\text {th }}$ RT ADCs were labeled as pre-ADC and intra-ADC, respectively.

\section{Statistical analysis}

The mean $\mathrm{ADC} \pm \mathrm{SD}$ of primary tumors including pre-ADC, pre-SD, intra-ADC, and intra-SD were acquired by each observer for each series of measurements. Secondly, inter- and intra-observer reproducibility of primary tumor ADC measurements tumor was determined by mean absolute difference (bias) and 95\% confidence interval of the mean difference (limits of agreement) according to the methods of Bland and Altman. BlandAltman plots were constructed by GraphPad-Prism 5 software. Statistical analyses were executed using SPSS 16.0 software (SPSS, Chicago, IL, USA), and the analysis of variance (ANOVA) was performed to compare the continuous variables between two groups.

\section{Abbreviations}

ADC: apparent diffused coefficient, DWI: diffusionweighted imaging, RT: radiotherapy, ROI: region of interest, ESCC: esophageal squamous cell carcinoma, PS: performance status.

\section{Author contributions}

Zhun Wang and Zhenfu Fu designed the study, Zhimin Ye and Jun Fang wrote the manuscript. Tieming Xie, Kai Li conducted MRI examination and ROI contouring, Shujun Dai collected the data and performed the analysis of statistics. Fangzheng Wang, and Yuezhen Wang supervised the analysis of imaging and assisted with manuscript preparation.

\section{CONFLICTS OF INTEREST}

On behalf of all authors of this paper, I declare that this study will not lead to any financial or other kinds of conflicts of interest. 


\section{FUNDING}

This work was supported by grants from Zhejiang Provincial Natural Science Funds (Grant Nos. LQ15H160012, LY17H160171) of China, the General Project of Zhejiang Provincial Health Bureau (Grant Nos.2015KYB063, 2015KYB048, 2016KYA051).

\section{REFERENCES}

1. Kandioler D, Schoppmann SF, Zwrtek R, Kappel S, Wolf B, Mittlböck M, Kührer I, Hejna M, Pluschnig U, Ba-Ssalamah A, Wrba F, Zacherl J. The biomarker TP53 divides patients with neoadjuvantly treated esophageal cancer into 2 subgroups with markedly different outcomes. A p53 Research Group study. J Thorac Cardiovasc Surg. 2014; 148:2280-6.

2. Katoh M, Igarashi M, Fukuda H, Nakagama H, Katoh M. Cancer genetics and genomics of human FOX family genes. Cancer Lett. 2013; 328:198-206.

3. Dutta S, Going JJ, Crumley AB, Mohammed Z, Orange C, Edwards J, Fullarton GM, Horgan PG, McMillan DC. The relationship between tumour necrosis, tumour proliferation, local and systemic inflammation, microvessel density and survival in patients undergoing potentially curative resection of oesophageal adenocarcinoma. $\mathrm{Br} \mathrm{J}$ Cancer. 2012; 106:702-10.

4. Minsky BD, Pajak TF, Ginsberg RJ, Pisansky TM, Martenson J, Komaki R, Okawara G, Rosenthal SA, Kelsen DP. INT 0123 (Radiation Therapy Oncology Group 94-05) phase III trial of combined-modality therapy for esophageal cancer: high-dose versus standard-dose radiation therapy. J Clin Oncol. 2002; 20:1167-74.

5. Oppedijk V, van der Gaast A, van Lanschot JJ, van Hagen P, van Os R, van Rij CM, van der Sangen MJ, Beukema JC, Rütten H, Spruit PH, Reinders JG, Richel DJ, van Berge Henegouwen MI, Hulshof MC. Patterns of recurrence after surgery alone versus preoperative chemoradiotherapy and surgery in the CROSS trials. J Clin Oncol. 2014; 32:385-91.

6. Baliyan V, Das CJ, Sharma R, Gupta AK. Diffusion weighted imaging: technique and applications. World J Radiol. 2016; 8:785-98.

7. Fujima N, Sakashita T, Homma A, Harada T, Shimizu Y, Tha KK, Kudo K, Shirato H. Non-invasive prediction of the tumor growth rate using advanced diffusion models in head and neck squamous cell carcinoma patients. Oncotarget. 2017; 8:33631-43. https://doi.org/10.18632/ oncotarget.16851.

8. Heijmen L, Verstappen MC, Ter Voert EE, Punt CJ, Oyen WJ, de Geus-Oei LF, Hermans JJ, Heerschap A, van Laarhoven HW. Tumour response prediction by diffusionweighted MR imaging: ready for clinical use? Crit Rev Oncol Hematol. 2012; 83:194-207.
9. Craciunescu OI, Yoo DS, Cleland E, Muradyan N, Carroll MD, MacFall JR, Barboriak DP, Brizel DM. Dynamic contrast-enhanced MRI in head-and-neck cancer: the impact of region of interest selection on the intra- and interpatient variability of pharmacokinetic parameters. Int $\mathrm{J}$ Radiat Oncol Biol Phys. 2012; 82:e345-50.

10. Wendl CM, Müller S, Eiglsperger J, Fellner C, Jung EM, Meier JK. Diffusion-weighted imaging in oral squamous cell carcinoma using 3 Tesla MRI: is there a chance for preoperative discrimination between benign and malignant lymph nodes in daily clinical routine?. Acta Radiol. 2016; 57:939-46.

11. van der Hoorn A, van Laar PJ, Holtman GA, Westerlaan HE. Diagnostic accuracy of magnetic resonance imaging techniques for treatment response evaluation in patients with head and neck tumors, a systematic review and metaanalysis. PLoS One. 2017; 12:e0177986.

12. De Cobelli F, Giganti F, Orsenigo E, Cellina M, Esposito A, Agostini G, Albarello L, Mazza E, Ambrosi A, Socci C, Staudacher C, Del Maschio A. Apparent diffusion coefficient modifications in assessing gastro-oesophageal cancer response to neoadjuvant treatment: comparison with tumour regression grade at histology. Eur Radiol. 2013; 23:2165-74.

13. Qiu B, Wang D, Yang H, Xie W, Liang Y, Cai P, Chen Z, Liu M, Fu J, Xie C, Liu H. Combined modalities of magnetic resonance imaging, endoscopy and computed tomography in the evaluation of tumor responses to definitive chemoradiotherapy in esophageal squamous cell carcinoma. Radiother Oncol. 2016; 121:239-45.

14. Wang L, Liu L, Han C, Liu S, Tian H, Li Z, Ren X, Shi G, Wang Q, Wang G. The diffusion-weighted magnetic resonance imaging (DWI) predicts the early response of esophageal squamous cell carcinoma to concurrent chemoradiotherapy. Radiother Oncol. 2016; 121:246-51.

15. Wang L, Han C, Zhu S, Shi G, Wang Q, Tian H, Kong $\mathrm{J}$, Zhang A. Investigation of using diffusion-weighted magnetic resonance imaging to evaluate the therapeutic effect of esophageal carcinoma treatment. Oncol Res Treat. 2014; 37:112-6.

16. Sakurada A, Takahara T, Kwee TC, Yamashita T, Nasu S, Horie T, Van Cauteren M, Imai Y. Diagnostic performance of diffusion-weighted magnetic resonance imaging in esophageal cancer. Eur Radiol. 2009; 19:1461-9.

17. Baer AH, Hoff BA, Srinivasan A, Galbán CJ, Mukherji SK. Feasibility analysis of the parametric response map as an early predictor of treatment efficacy in head and neck cancer. AJNR Am J Neuroradiol. 2015; 36:757-62.

18. Hwang I, Choi SH, Kim YJ, Lee AL, Yun TJ, Kim JH, Kim KG, Sohn CH. Differentiation of recurrent tumor and posttreatment changes in head and neck squamous cell carcinoma: application of high b-value diffusion-weighted imaging. AJNR Am J Neuroradiol. 2013; 34:2343-8.

19. Sun YS, Cui Y, Tang L, Qi LP, Wang N, Zhang XY, Cao K, Zhang XP. Early evaluation of cancer response by a new 
functional biomarker: apparent diffusion coefficient. AJR Am J Roentgenol. 2011; 197:W23-9.

20. Vandecaveye V, Dirix P, De Keyzer F, Op de Beeck K, Vander Poorten V, Hauben E, Lambrecht M, Nuyts S, Hermans R. Diffusion-weighted magnetic resonance imaging early after chemoradiotherapy to monitor treatment response in head-and-neck squamous cell carcinoma. Int J Radiat Oncol Biol Phys. 2012; 82:1098-107.
21. Kwee RM, Dik AK, Sosef MN, Berendsen RC, Sassen $\mathrm{S}$, Lammering G, Clarijs R, Oostenbrug LE, Blom RL, Vliegen RF. Interobserver reproducibility of diffusionweighted MRI in monitoring tumor response to neoadjuvant therapy in esophageal cancer. PLoS One. 2014: 9:e92211.

22. Kwee TC, Takahara T, Luijten PR, Nievelstein RA. ADC measurements of lymph nodes: inter- and intra-observer reproducibility study and an overview of the literature. Eur J Radiol. 2010; 75:215-20. 